\title{
The Influences of Cognitive Psychology of Interpreting on Interpreter Training: An Empirical Study on Interpreting Anxiety of Student Interpreters
}

\author{
Lu Wang ${ }^{1, a}$, Ming-Tsang, Hsieh ${ }^{1, b,{ }^{*}}$
}

${ }^{1}$ School of Foreign Languages and International Trade, Wenzhou Business College, China

awanglu_2016@163.com, bxmc@wzbc.edu.cn

${ }^{*}$ Corresponding author

\begin{abstract}
Keywords: Self-schema of interpreting anxiety; Interpreting flow; Interpreting quality; Structural
\end{abstract} equation model; Empirical study.

\begin{abstract}
Based on self-schema and flow theory, this study investigates the relationship among self-schema of interpreting anxiety (SIA), interpreting flow (IF) and interpreting quality (IQ). Hybrid research methods are adopted is this study, including questionnaire and semi-structured interview. A structural equation model is developed to verify the moderating effect of interpreting anxiety (IA) and test the casual effects among those constructs to explore the cognitive process and the source and effect of IA. The empirical results show that (1) SIA has a significant positive effect on IF;(2) IF has a significant positive effect on IA;(3) Student interpreters of different IA degrees show significant differences in the relationship among SIA, IF and IQ;(4) For student interpreters, the sources of IA mainly derive from a lack of confidence and interpreting skills and concerns for the environment of interpreting. Based on the empirical results, implications for interpreting training of student interpreters are proposed, including construction of SIA, arousing IF, building self confidence in order to improve IQ.
\end{abstract}

\section{Introduction}

The development of professionalization and specialization of interpreting in the new century has come to point where the interpreter's psychological state is worth examining. Spurred by flourishing development of interdisciplinary research, theories and data analysis methods borrowed from related disciplines have attracted much attention in the study of cognitive process of interpreting over the past few decades. Interpreters are exposed to many uncertainties including accent, terminology, noise, as well as audience's feedback. Moreover, immediacy and multi-tasking process of interpreting will also cause anxiety for both student and professional interpreters [1]. Kang (2012) claimed that IA is influential with IQ, and different degrees of IA would generate varied performance [2]. Kang also pointed out that medium anxiety would active certain brain cells in linguistic area and enhance IQ. Many scholars have conducted researches regarding the influence of IA on interpreting and unveiled the interactive relation between them. Though theoretical and empirical studies regarding to interpreting cognition conducted before are many, an empirical research particularly centering on the formation, prevention, intervention of IA is quite few. Therefore, this study will discuss the relation among SIA, IF, IQ by applying self-schema theory and flow theory, a mixed approach with questionnaire and semi-structured interview and building "SIA-flow" mode to further testify the moderating effect and source and influence of IA.

\section{Literature review and hypotheses}

\subsection{Self-schema of interpreting anxiety}

Self-schema of interpreting is a general cognition of one's own interpreting skills which is based on one's interpreting experience. Generally speaking, interpreters process information on the basis of self-schema of interpreting. Interpreters may memorize meaningful and relative knowledge of interpreting with the help of self-schema which serves as effective reference and example for the 
future interpreting $[1,3]$. However, SIA is a general cognition of IA which is built on interpreter's capability and experience of ways to cope with anxiety. On the basis of SIA, interpreters can seek appropriate ways to overcome anxiety and enhance IQ [4, 5]. Previous studies indicated that evaluation of SIA should include cognition, emotion and behavior, which are the main research contents in this study $[4,5]$.

\subsection{Interpreting cognition, emotion and behavior evaluations}

Rui and JI (2017) believed that self-cognition evaluation is a formation based on interpreter's self-efficacy cognition. If interpreters hold weak faith in IQ, then it can be called self-efficacy in interpreting [5]. Chen et al. (2001) mentioned that self-efficacy referred to low self-cognition that one's believing in its personal competence such as motivation, cognitive resources and course of action in a particular circumstance [6]. The studies also indicated that proper self-efficacy not only varies one's goal attainment capability, but also leads to the transformation and restructure of interpreter's cognitive beliefs, and such circumstance may reflect differently in different functional domains [6].

Emotion is a functional mode evolved from systematic development of human being and it serves for certain adaptive functions, such as reproduction, subordinate relation, mastering and learning and so on. These functions are also evoked by certain circumstances. Viewed from the concept of multi-componential system, emotion combination is diversified, including cognitive patterns, motivation trends, expression trends and independent self-activation [7]. Moreover, plenty factors would affect emotion building and the combination is quite complex. Therefore, every impressive memory that people cared about may deeply rooted and embodies because of varied factors [7]. For student interpreters, the emotions produced in the course of interpreting affect their attitudes and motivation for future interpreting learning. Student interpreters who usually have positive emotions may enjoy learning or working as an interpreter.

Carver and White (1994) believed that physiological mechanism, also known as the behavioral activation system, controls the motivation of desires [8]. This mechanism is responsible for positive emotion experience such as hope, joy, excitement, happiness and so on, and highly sensitive to reward, evasion and punitive and non-punitive signals. Rui and JI (2017) described that for student interpreter their positive or negative attitude towards interpreting determine the results of interpreting evaluation [5]. Under the circumstance of low pro-activeness, student interpreter may unlikely to conduct positive learning and vice versa.

\subsection{Interpreting flow}

Landers et al. (2015) and Koufaris (2002) believed that flow is an emotional indicator describing how participant feel in certain activities $[9,10]$. When the flow is produced, one may feel immersed in the activity, weaken the focus of self-awareness and filter irrelevant consciousness and thoughts. Then, as the self-consciousness receding, participants will produce clear pursuit of goals and definite response, and feel the entire circumstance is under control. Robinson (2003) had described that there is a state called "automatic translation" happened both in translation and interpreting verifying the applicability of flow theory [11]. Landers et al. (2015) also pointed out that flow experience would be more helpful when interpreter or translator feel qualified for the task, especially challenging tasks [9]. A translator or interpreter can easily enter into flow experience if he is passionate about the task, filter irrelevant information and thoughts consciously, and concentrate on conduct the task with a feeling of control in the whole process. Based on this, ths study here propose: H1: ISE positively influences the forming of IF. H2: IEE of positively influences the forming of IF. H3: IBE positively influences the forming of IF.

\subsection{Interpreting quality}

Interpreting quality (IQ) is an indicator suggesting how well an interpreting is delivered. However, IQ consists of complex components, including output of target language, delivery of meaning, audience feedback and communicative effect [12]. As the sole bilingual person knowing the purposes of two parties, interpreter should attach great importance to these factors. Also, 
self-monitor system and audience feedback are convenient for interpreters to conduct self-evaluation. Therefore, self-evaluation acts as an important part to successful delivery of target language and improve interpreting skills. Harmat et al. (2015) believed that the flow helps interpreter feel relax and stay focused in the task so as to improve IQ [13]. Thus, this study posits:H4: IF positively influences IQ.

\subsection{Interpreting anxiety}

Dong et al. (2013) claimed that anxiety exists in linguistic learning, especially interpreting learning [1]. Kang (2011) defined that IA is a result of multiple factors, such as immediacy of interpreting, complexity of source language, similarities and dissimilarities between source and target language and abnormal emotional factors [3].This study defines the IA as a temporarily unease state caused by factors such as immediacy of interpreting, complexity of source language, similarities and dissimilarities between source and target languages and other unusual emotional factors according to the studies of Dong et al. (2013), Kang (2011) and Spilberger and Gorsuch (1983) [1, 3, 14]. Kang (2012) believed that different degrees of IA would bring student interpreters with positive or negative influences [2]. Medium IA (temperate anxiety) serves as catalyst for interpreting performance while high IA or low IA both cause negative effects [3]. Therefore, the study here proposes: H5: Compared with high IA or low IA, constructs in the conceptual model are closely related when interpreters are in medium IA. Based on the above hypotheses, a conceptual model is established as follows (Fig. 1).

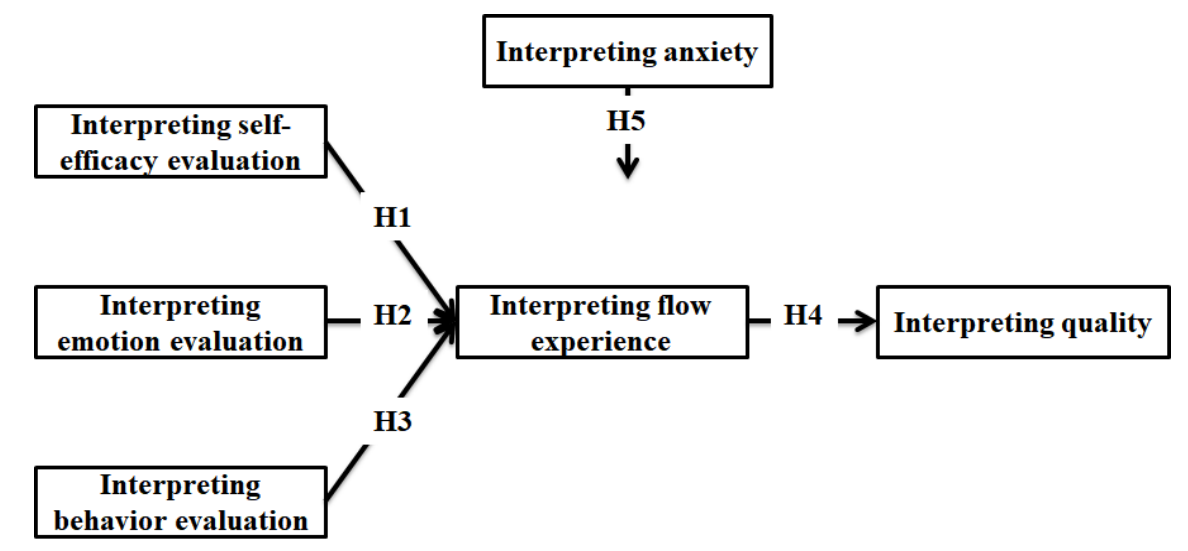

Fig. 1. Conceptual model

\section{Methods}

\subsection{Questionnaire and pre-test}

The questionnaire is composed of 6 parts. Part 1 is designed to investigate the interpreting experience of student interpreters and the next three parts are indicators to measure several constructs by using seven-point likert scale. The last part is focused on information of subjects. All of the Cronbach's alpha of each construct in pre-test are greater than 0.7 , showing high internal consistency [15].

Table 1 . The reliability of pre-test and references of items

\begin{tabular}{|c|c|c|c|c|}
\hline \multicolumn{2}{|c|}{ Construct } & Items & Cronbach's alpha & References \\
\hline \multirow{3}{*}{ SIA } & ISE & 8 & 0.846 & \multirow{3}{*}[6,7,8]{} \\
\hline & IEE & 9 & 0.899 & \\
\hline & IBE & 4 & 0.804 & \\
\hline \multirow{5}{*}{ IF } & Concentration & 4 & 0.805 & \multirow{3}{*}[9,10]{} \\
\hline & Perceived Control & 5 & 0.916 & \\
\hline & Enjoyment & 5 & 0.846 & \\
\hline & IQ & 12 & 0.915 & [16] \\
\hline & IA & 18 & 0.883 & [3] \\
\hline
\end{tabular}




\subsection{Data collection and sample profile}

Samples refer to undergraduate or postgraduate who have received interpreting training and have interpreting experience in the last 6 months. Total of 265 questionnaires were distributed, and 205 valid responses were returned (77.4\%). Among the respondents, 40 students were male (19.5\%) while 165 were female $(80.5 \%)$ and the age ranges from 21 to 30 years. As for their education level, undergraduates account for $82.4 \%$ and postgraduates $17.6 \%$.

\section{Results}

This study used ADANCO 2.0 for the analysis of measurement model and structural model and the comparison and contrast of these models.

\subsection{Measurement model}

As illustrated in Table 2, internal consistency reliability was established for all reflective constructs as Cronbach's $\alpha$ and composite reliability (rho A) estimates were greater than $0.8[15,17]$; and the AVE of all the unidimensional constructs are greater than 0.5 revealing appropriate convergent validity [18]. Furthermore, the HTMT values significantly lower than 1 and show discriminant validity requirements [19]. Next, this study verified the goodness of model fit via overall model assessment. Henseler et al. suggested that the HI95 of SRMR, duLs and $\mathrm{d}_{\mathrm{G}}$, should be higher than the original values and the approximate model fit should be lower than 0.08 [19]. Thus, the goodness of model fit in this study does not meet all of the criteria but is still acceptable.

Table 2 . The reliability and validity analysis for the constructs

\begin{tabular}{ccccc}
\hline Construct & Items & Cronbach' $\alpha$ & rho A & AVE \\
\hline ISE & 8 & 0.940 & 0.945 & 0.709 \\
IEE & 9 & 0.955 & 0.960 & 0.738 \\
IBE & 4 & 0.877 & 0.890 & 0.728 \\
IF & 14 & 0.958 & 0.964 & (Not unidimensional) \\
Concentration & 4 & 0.865 & 0.899 & 0.704 \\
Perceived Control & 5 & 0.948 & 0.950 & 0.830 \\
Enjoyment & 5 & 0.958 & 0.958 & 0.855 \\
IQ & 12 & 0.963 & 0.968 & 0.719 \\
IA & 18 & 0.954 & 0.959 & 0.569 \\
\hline
\end{tabular}

\subsection{Structural model and tests of hypotheses}

Structural model showed that the former four hypotheses are fully supported and verified the fact that high level of ISE, IEE and IBE would be conducive to IF and there were positive correlations between IF and IQ. These results fully indicate the importance of the SIA for student interpreters.

Table 3 Assessing the hierarchical construct models, structural model and hypothesis testing

\begin{tabular}{|c|c|c|c|c|}
\hline Path & Coefficient (Standard error) & t-value & Sig. & Cohen's $\mathrm{f}^{2}$ \\
\hline \multicolumn{5}{|l|}{$\begin{array}{l}\text { Second-order model } \\
\text { IF }\end{array}$} \\
\hline Concentration & $0.892^{* * *}(0.013)$ & 68.884 & 0.000 & 3.898 \\
\hline Perceived Control & $0.884^{* * *}(0.016)$ & 55.016 & 0.000 & 3.590 \\
\hline Enjoyment & $0.943^{* * *}(0.007)$ & 132.586 & 0.000 & 8.045 \\
\hline \multicolumn{5}{|l|}{ Structural Model } \\
\hline $\mathrm{ISE} \rightarrow \mathrm{IF}(\mathrm{H} 1)$ & $0.214^{* * *}(0.052)$ & 4.098 & 0.000 & 0.112 \\
\hline $\mathrm{IEE} \rightarrow \mathrm{IF}(\mathrm{H} 2)$ & $0.550^{* * *}(0.053)$ & 10.386 & 0.000 & 0.574 \\
\hline $\mathrm{IBE} \rightarrow \mathrm{IF}(\mathrm{H} 3)$ & $0.235^{* * *}(0.054)$ & 4.355 & 0.000 & 0.158 \\
\hline $\mathrm{IF} \rightarrow \mathrm{IQ}(\mathrm{H} 4)$ & $0.840^{* * *}(0.021)$ & 39.671 & 0.000 & 2.389 \\
\hline$R^{2}$ & \multicolumn{4}{|c|}{0.705} \\
\hline Adjusted $R^{2}$ & \multicolumn{4}{|c|}{0.704} \\
\hline
\end{tabular}




\subsection{Testing of moderating effect}

This study divided the subjects into three groups, high (IA $>90)$, medium $(72<$ IA $<92)$ and low $(\mathrm{IA}<72)$. Through the independent sample $\mathrm{T}$ test, there is a significant difference in the mean value between these three groups.

Table 4 The result of test of moderating effects of IA (high vs medium)

\begin{tabular}{cccccc}
\hline Path $/$ Models & High $(\mathrm{N}=71)$ Coefficient (S.E.) & Medium $(\mathrm{N}=65)$ Coefficient (S.E.) & Difference & $\mathrm{t}$-value & Sig. \\
\hline $\mathrm{ISE} \rightarrow \mathrm{IF}$ & $0.137(0.101)$ & $0.060(0.073)$ & 0.077 & 0.613 & 0.541 \\
$\mathrm{IEE} \rightarrow \mathrm{IF}$ & $0.637^{* * *}(0.127$ & $0.560^{* * *}(0.089$ & 0.077 & 0.492 & 0.624 \\
$\mathrm{IBE} \rightarrow \mathrm{IF}$ & $0.219^{*}(0.108)$ & $0.361^{* * *}(0.106)$ & 0.142 & 0.943 & 0.348 \\
$\mathrm{IF} \rightarrow \mathrm{IQ}$ & $0.966^{* * *}(0.006)$ & $0.797^{* * *}(0.048)$ & 0.169 & 3.676 & 0.000 \\
\hline$R^{2}$ & 0.933 & 0.636 & --- & --- & --- \\
Adjusted $R^{2}$ & 0.932 & 0.630 & --- & --- & --- \\
\hline${ }^{*} p<0.05,{ }^{* *} p<0.01,{ }^{* * *} p<0.001$ & & & &
\end{tabular}

This analysis shows different path coefficients of the three groups. As is shown in the table, H5 is partially supported and the positive effect of path "IBE $\rightarrow$ IF" is only significant in medium IA group.

Table 5 The result of test of moderating effects of IA (medium vs low)

\begin{tabular}{cccccc}
\hline Path $/$ Models & Medium $(\mathrm{N}=65)$ Coefficient (S.E.) & Low $(\mathrm{N}=69)$ Coefficient (S.E.) & Difference & t-value & Sig. \\
\hline $\mathrm{ISE} \rightarrow \mathrm{IF}$ & $0.060(0.073)$ & $0.384^{* * *}(0.085)$ & 0.324 & 2.898 & 0.004 \\
$\mathrm{IEE} \rightarrow \mathrm{IF}$ & $0.560^{* * *}(0.089$ & $0.494^{* * *}(0.096)$ & 0.066 & 0.506 & 0.613 \\
$\mathrm{IBE} \rightarrow \mathrm{IF}$ & $0.361^{* * *}(0.106)$ & $0.144(0.084)$ & --- & --- & --- \\
$\mathrm{IF} \rightarrow \mathrm{IQ}$ & $0.797^{* * *}(0.048)$ & $0.826^{* * *}(0.035)$ & 0.029 & 0.496 & 0.621 \\
\hline$R^{2}$ & 0.636 & 0.683 & --- & --- & --- \\
Adjusted $R^{2}$ & 0.630 & 0.678 & --- & --- & --- \\
\hline${ }^{*} p<0.05,{ }^{* *} p<0.01,{ }^{* * *} p<0.001$ & & & &
\end{tabular}

\subsection{Qualitative interview}

Semi-structured interviews are used in the conduction of qualitative research.According to the interviews of 65 student interpreters, unfamiliar topic, insufficient accumulation of terminology, lack of skills in listening and comprehension form the prominent factors of IA.Moreover, lack of background knowledge, real interpreting experience and self-confidence act as the main obstacles on their ways to be professional interpreters.

\section{Conclusion}

The main aim of this research was to examine the moderating effect of IA in the structural model. The study carried out showed that, in the case of student interpreters, SIA has a significant positive effect on IF, IF has a significant positive effect on IQ and different degrees of IA show significant differences in the relationship among self-schema of IA, IF and IQ. This study makes both theoretical and practical contributions. At theoretical level, this study offers a model for the analysis of IA, empirically tested, of the consequences of student interpreters. Furthermore, this study investigated the link among SIA, IF and IQ. At a practical level, this study points out that interpreting teachers are suggested to adopt differentiated teaching method in the light of different degrees of IA and seek corresponded training method according to the effects of IBE, IEE and ISE to help student interpreters enter into IF and improve IQ. Care should be taken in interpreting training, learning and instructional evaluation based on the result of this study. Firstly, interpreting teachers should give emphasis to strengthen of SIA and importance of IF. Mock conference interpreting can be regarded as an effective way to increase student interpreters' cognitive presence and be conducive to communicative competence and evaluation of SIA. Secondly, flexible adjustments can also be taken in the process of training, including the choosing of training material, ways of guidance, remarks on their performance and even the arrangement of seat in the classroom. Teachers may divide the students into three individual learning groups, namely, "active group", 
"interactive group" and "self-directed group", thus, students are given the very space to interact with teachers or not to regulate IA by themselves. Finally, based on the multimedia teaching equipment in the classroom, teachers and student interpreters can make full use of the advantages of Internet to realize resource sharing, including create social groups, share course ware and practical interpreting training videos in order to create a simulation environment for students to improve interpreting skills and adjusting IA anytime and anywhere.

\section{References}

[1] Y. P. Dong, H. P. Chen and J. B. Yu, Developing an interpreting anxiety scale, Foreign Language World, Vol. 6, pp. 57-64, 2013.

[2] Z. F. Kang, Interpreting anxiety with multimodalities: The sources of degrees, Foreign Language Education, Vol. 33, pp. 106-109, 2012.

[3] Z. F. Kang, The causations, the degrees and the impacts of interpreting anxiety, Foreign language research, Vol. 4, pp. 81-85, 2011.

[4] J. H. Wang, Multifactor evaluations on how interpretation learner's motive influences their performance, Foreign language research, Vol. 3, pp. 93-97, 2014.

[5] Y. P. Rui and H. J. Ji, The impact of multimodal listening \& speaking teaching on English speaking anxiety and classroom reticence, Computer-assisted Foreign Language Education, Vol. 6, pp. 50-55, 2017.

[6] G. Chen, S. M. Gully and D. Eden, Validation of a new general self-efficacy scale, Organizational Research Methods, Vol. 4, pp. 62-83, 2001.

[7] E. M. Tong and L. Jia, Positive emotion, appraisal, and the role of appraisal overlap in positive emotion co-occurrence, Vol. 17, pp. 40-54, 2016.

[8] C. S. Carver and T. L. White, Behavioral inhibition, behavioral activation, and affective responses to impending reward and punishment: The BIS/BAS Scales, Journal of Personality and Social Psychology, Vol. 67, pp. 319-333, 1994.

[9] V. M. Landers, S. E. Beatty, S. Wang and D. L. Mothersbaugh, The effect of online versus offline retailer-brand image incongruity on the flow experience, Journal of Marketing Theory \& Practice, Vol. 23, pp. 370-387, 2015.

[10]M. Koufaris, Applying the technology acceptance model and flow theory to online consumer behavior, Informs, 2002.

[11]F. Robinson, Latest news from flair - flow Europe 4, Nutrition Bulletin, Vol. 28, pp. 59-64, 2010.

[12]X. H. Cai and F. Q. Fang, Evaluation interpreting quality and effect, Foreign Languages and Their Teaching, Vol. 3, pp. 41-45, 2003.

[13]L. Harmat, M. O. De, T. Theorell, L. Högman, H. Fischer and F. Ullén, Physiological correlates of the flow experience during computer game playing, International Journal of Psychophysiology, Vol. 97, pp. 1-7, 2015.

[14]C. D. Spielberger and R. L. Gorsuch, Manual for state-trait anxiety inventory (Form Y), Consulting Psychologists Press, 1983.

[15]M. L. Roberts and L. H. Wortzel, New life-style determinants of women's food shopping behavior, Journal of Marketing, Vol. 43, pp. 28-39, 1979.

[16]T. K. Dijkstra and J. Henseler, Consistent partial least squares path modelling, MIS Quarterly, Vol. 39, pp. 297-316, 2015. 
[17]X. H. Cai, Interpretation evaluation (original version), China translation Corporation, 2007.

[18]J. C. Anderson and D. W. Gerbing, Structural equation modeling in practice: A review and recommended two-step approach, Psychological Bulletin, Vol. 103, pp. 411-423, 1988.

[19]J. Henseler, G. Hubona and P. A. Ray, Using PLS path modeling in new technology research: updated guidelines, Industrial Management \& Data Systems, Vol. 116, pp. 2-20, 2016. 